\title{
A Universal Interface for Video Game Machines Using Biological Signals
}

\author{
Keisuke Shima, Nan Bu, Masaru Okamoto, and Toshio Tsuji \\ Graduate School of Engineering, Hiroshima University, Japan \\ \{keisuke, bu, okamoto, tsuji\}@bsys.hiroshima-u.ac.jp \\ http://www.bsys.hiroshima-u.ac.jp
}

\begin{abstract}
This paper proposes a universal entertainment interface for operation of amusement machines, such as video game machines and radio control toys. In the proposed interface system, biological signals are used as input, where users can choose some specific biological signal and configuration of signal measurement in accordance with their preference, physical condition (disabled or not), and degree of the disability. From the input signals, users' intention of operation can be estimated with a probabilistic neural network (PNN), and then, control commands can be determined accordingly. With the proposed interface, people, even those with severe physical disabilities, are able to operate amusement machines. To verify validity of the proposed method, experiments were conducted with a video game machine.
\end{abstract}

\section{Introduction}

Recently, a variety of amusement machines, such as video game machines, radio control toys, and entertainment robots, have been rapidly developed, and has found wide application in people's daily lives. It has been confirmed that amusement machines can be used as a method of rehabilitation therapy for people with physical disabilities [1]-[3]. However, operation of amusement machines might be difficult for the disabled, since movements of the users' body are necessary to operate input devices, such as switches and buttons. To deal with this problem, universal entertainment interfaces are required, with which even people with severe physical disabilities can enjoy amusement machines.

In the field of entertainment interfaces, a lot of research has been carried out to enable people with physical disability to operate amusement machines 4, 5 . Most of this research attempts to design dedicated input devices according to the users' physical condition and their capability for controlling the movements of the head, feet, hands, and other parts of the body. An interface for Play Station 2 (PS2) was proposed by replacing PS2's controller with large buttons or the use of shifting body weight for operation [4]. Also, according to the INTERCOMUNICANDO project, games can be controlled using various switches (hand operated, foot operated, and so on), which can be chosen depending on the users' capability of body movement [5]. However, these interfaces require

F. Kishino et al. (Eds.): ICEC 2005, LNCS 3711, pp. 88-98 2005.

(C) IFIP International Federation for Information Processing 2005 
some (specific) body movements, which are difficult for people with severe physical disabilities, such as patients who have cervical spine injuries and those with amyotrophic lateral sclerosis.

On the other hand, in the literature of human machine interfaces, systems using biological signals have been intensly studied [6], [7]. Krepki et al. proposed a brain computer interface as a pointing device of computers, and this system can control simple computer games based on the users' intention estimated from electroencephalogram (EEG) signals [6]. Also, Betke et al. proposed a visionbased human-computer interface. This system tracks users' movements with a video camera and translates them into the movements of a mouse pointer on screen, and it has been successfully applied to several computer games [7]. However, these interfaces have not been applied to other amusement machines, e.g., video games. Also, it has been widely accepted that users have to take a longterm training process to gain dexterous skill for operation, because estimation of the users' intention from EEG features is extremely difficult.

In this paper, a novel entertainment interface is proposed, and operation of video game machines is presented to illustrate validity of the proposed method. With this system, users can choose some specific biological signals and measurement configuration with respect to their preference and degree of physical disabilities. The users' intention of operation is estimated from the biological signals using a probability neural network (PNN), and the differences in biological signals among individuals can be covered by using adaptive learning. Then, operation commands are set according to the users' physical conditions and the selected video game. Also, many kinds of video game machines can be incorporated, since the control and communication functions are implemented on a reconfigurable hardware, field programmable gate array (FPGA).

This paper is organized as follows: In Section 2, the proposed entertainment interface system and a prototype are described. Section 3 presents experimental results of healthy subjects and a patient with a cervical spine injury. Finally, Section 4 concludes this paper.

\section{A Universal Entertainment Interface}

The proposed universal entertainment interface system is shown in Fig. 1, This system consists of four parts: (1) signal measurement, (2) operation estimation, (3) command encoding, and (4) signal conversion. In this section, a prototype of the proposed system is introduced.

\subsection{Signal Measurement}

A variety of biological signals such as electromyogram (EMG) signals and EEG signals can be used to extract the intention of operation and to detect motions of the human body. These signals reflect the physiological information on movement and the internal state of users. Also, movements of the body can be detected from acceleration (ACC) signals. In the proposed method, users can choose some specific biological signals and measurement configuration according 


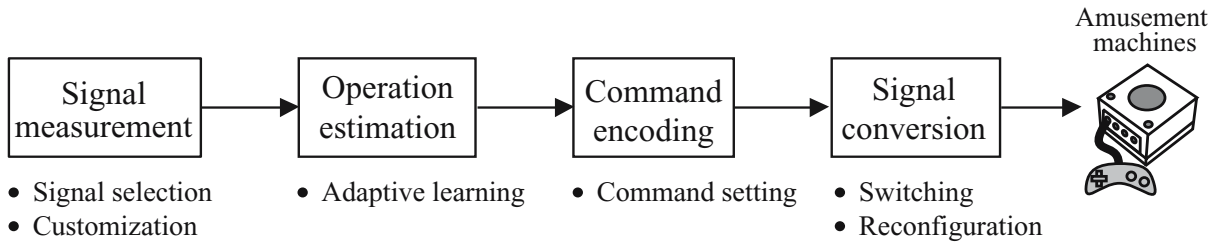

Fig. 1. The proposed entertainment interface system

to their preferences and degree of the disability. Because of this flexible choice of input signals, it is expected that the number of potential users of amusement machines would greatly increase.

\subsection{Operation Estimation}

In this part, probabilistic neural networks (PNNs) are used for discrimination of operation from biological features. In the context of pattern discrimination, a large amount of research has been carried out using PNNs [9-[13]. It is widely accepted that, due to the prominent nonlinear approximation capability, PNNs can estimate the posterior probability distribution of input patterns with arbitrary accuracy by training the network architecture and the weights appropriately. So far, PNNs have been used as an important tool for pattern discrimination, and have been proven to be efficient especially for complicated problems such as discrimination of biological signals [12, [13].

Features extracted from biological signals are inputted into PNNs. Let us take EMG signals for example. $L$ pairs of $\mathrm{Ag} / \mathrm{AgCl}$ electrodes with conductive paste are attached to muscles. The $L$ channels of EMG signals inputted are rectified and filtered by a second-order Butterworth filter. The filtered EMG signals are defined as $E M G_{l}(t)(l=1, \cdots, L)$. Then, these EMG signals are normalized to make the sum of $L$ channels equal 1 :

$$
x_{l}(t)=\frac{E M G_{l}(t)-E M G_{l}^{s t}}{\sum_{l^{\prime}=1}^{L}\left(E M G_{l^{\prime}}(t)-E M G_{l^{\prime}}^{s t}\right)}
$$

where $E M G_{l}^{s t}(t)$ is the mean value of $E M G_{l}(t)$ which is measured while relaxing the muscles. Feature vector $\boldsymbol{x}(t)=\left[x_{1}(t), x_{2}(t), \cdots, x_{L}(t)\right]^{\mathrm{T}}$ is used for pattern discrimination.

In the prototype system, a log-linearized Gaussian mixture network (LLGNM) [12 is used. LLGMN is based on a Gaussian mixture model (GMM) and a log-linear model of probability distribution function (pdf). By applying the log-linear model to a product of the mixture coefficients and the mixture components of GMM, a semiparametric model of pdf is incorporated into a three-layer feedforward NN, as shown in Fig. 2. For details of LLGMN, please refer to [12. LLGMN estimates probability distribution from the input vector $\boldsymbol{x}(t)$, and outputs posterior probability of operation $k(k=1, \ldots, K)$. 


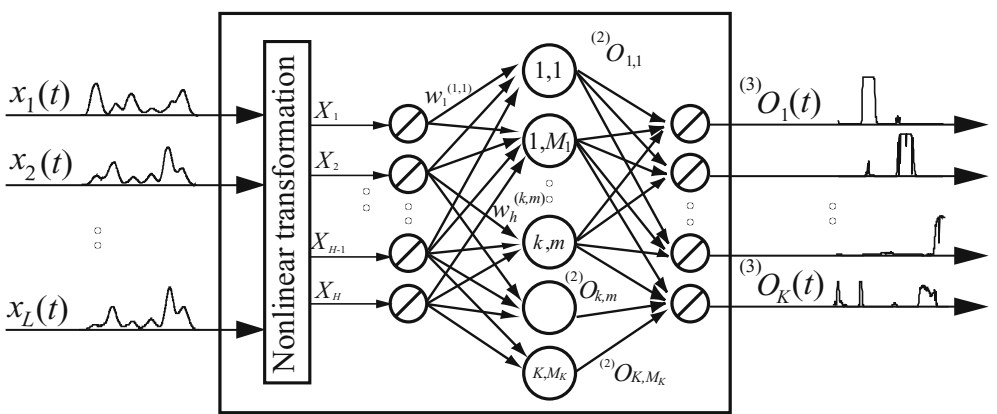

Fig. 2. EMG pattern discrimination using LLGMN

To determine when the operation occurs, force information $F_{E M G}(t)$ is calculated from EMG signals,

$$
F_{E M G}(t)=\frac{1}{L} \sum_{l=1}^{L} \frac{E M G_{l}(t)-E M G_{l}^{s t}(t)}{E M G_{l}^{a v g}(t)-E M G_{l}^{s t}(t)}
$$

where $E M G_{l}^{a v g}(t)$ is the mean value of $E M G_{l}(t)$ measured under an average contraction level that is determined by the user. Since we plan to integrate the signal processing part into a hardware platform, the power level $P L(t)$ is set as $[0,1]$ to simplify the data representation:

$$
P L(t)= \begin{cases}F_{E M G}(t) & \left(F_{E M G}(t) \leq 1\right) \\ 1 & \left(F_{E M G}(t)>1\right)\end{cases}
$$

Then, $P L(t)$ is compared with a threshold $M_{d}$. When $P L(t)$ is more than $M_{d}$, intention of operation would be discriminated. In addition, entropy of the output of LLGMN is used to avoid misdiscrimination [14. The entropy is defined as

$$
E(t)=-\sum_{k=1}^{K}{ }^{(3)} O_{k}(t) \log ^{(3)} O_{k}(t)
$$

where $O_{k}(t)$ indicates the posterior probability of operation $k$. If $E(t)$ is less than a threshold $E_{d}$, an operation, which has the largest posterior probability, is determined. In contrary, when $E(t)$ is more than $E_{d}$, discrimination should be suspended, since large entropy suggests that the network's output is ambiguous.

\subsection{Command Encoding}

In the step of command encoding, commands of amusement machines are encoded based on the estimated operations. The prototype system uses video games as controlled objects. Because video games differ in the numbers of commands and the response speed required for operation, different protocols are needed for different kinds of games. In the proposed method, a direct mapping or a 
Table 1. Classification of the video games

\begin{tabular}{|c|c|c|c|}
\hline & & \multicolumn{2}{|c|}{ Number of commands } \\
\hline & & Large & Small \\
\hline \multirow[t]{2}{*}{$\begin{array}{c}\text { Response } \\
\text { speed }\end{array}$} & Quick & $\begin{array}{l}\text { - Fighting } \\
\text { - Rhythm } \\
\text { - First-person shooting } \\
\text { - Sports } \\
\text { - Action } \\
\text { - Action role playing } \\
\text { - Real-time simulation }\end{array}$ & $\begin{array}{l}\text { - Racing } \\
\text { - Shooting } \\
\text { - Tetris type }\end{array}$ \\
\hline & Slow & $\begin{array}{l}\text { - Role playing } \\
\text { - Simulation role playing } \\
\text { - Simulation }\end{array}$ & $\begin{array}{l}\text { - Puzzle } \\
\text { - Table } \\
\text { - Board } \\
\text { - Adventure }\end{array}$ \\
\hline
\end{tabular}

shift-group method is used. The direct mapping assigns each operation to a different command. For the shift-group protocol, commands are divided into several groups. In each group, operations are directly mapped into commands. By shifting these groups, objective commands can be achieved, with a smaller number of operations.

In this paper, games are classified according to response speed of operation and the numbers of commands (see Table 1). In Table 11 games with a large number of commands and quick response speed are classified as Type 1. Similarly, definitions of the other types are given as: Type 2 (small-quick), Type 3 (large-slow), and Type 4 (small-slow). For games of Type 3, to perform all the commands with a limited a number of operations, the shift-group protocol can be applied. In the case of Type 4 games, direct mapping can be used. However, patients with severe physical disabilities may conduct very small number of operations, say just one or two. For such users, the shift-group protocol is applicable.

In addition, the command encoding part uses an operation threshold $\alpha$ to avoid errors of operation. Given the number of consecutive estimations of the same operation $\left(R_{\text {count }}\right)$, the operation is discriminated when $R_{\text {count }}$ exceeds $\alpha$.

\subsection{Signal Conversion}

In the signal conversion, the control signals are determined based on commands, and then transmitted to the target game machine following communication protocols. However, since communication protocols vary largely among amusement machines, the signal conversion part needs to be reconstructed whenever the game machine is changed. In the proposed interface system, a reconfigurable hardware is used as an implementation platform for signal conversion. In the prototype system, a field programmable gate array (FPGA) chip is used. FPGA 


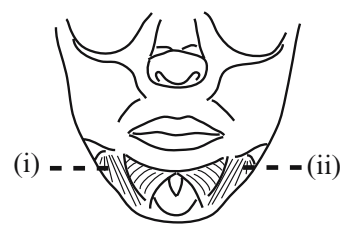

(i) Right Depressor anguli oris

(ii) Left Depressor anguli oris

(a) Mimetic muscles (Depressor anguli oris)

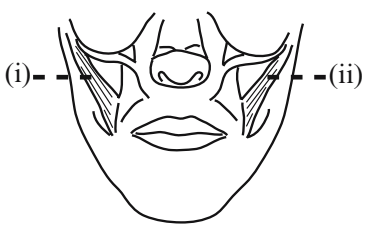

(i) Right Zygomaticus major

(ii) Left Zygomaticus major

(b) Mimetic muscles (Zygomaticus major)

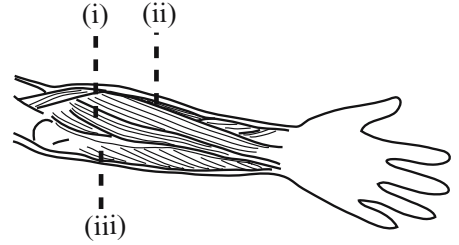

(i) Extensor Carpi Ulnaris

(ii) Extensor Carpi Radialis

(iii) Flexor Carpi Ulnaris

(c) Forearm muscles

Fig. 3. Locations of electrodes

is chosen since it allows easy reprogrammability, fast development times, and reduced efforts with respect to large-scale integration (LSI) design. When the game machine is changed, FPGA can be reconfigured at will. Consequently, a variety of amusement machines can be operated with a single hardware device.

\section{$3 \quad$ Experiments}

To examine validity of the proposed method, EMG discrimination experiments and video game operation experiments using PS2 were performed with five subjects (A, B, C, and D: healthy people, E: a patient with a cervical spine injury).

\subsection{Experimental Condition}

In the experiments, electrodes were attached to the following three different locations: (a) depressor anguli oris muscles ( $L=2$; on the right and left sides), and (b) zygomaticus major muscles ( $L=2$; on the right and left sides), (c) forearm muscles ( $L=4$; right hand: extensor carpi ulnaris, extensor carpi radialis, and flexor carpi ulnaris; left hand: extensor carpi ulnaris), as shown in Fig. 3 For subject E, operation with forearm muscles was not conducted, because his arms are paralyzed. The EMG signals after rectification and smoothing were digitized using the 8bit $\mathrm{AD}$ converter with a sampling frequency of $50 \mathrm{~Hz}$. The feature pattern, $\boldsymbol{x}(t)$, was used to train LLGMN. In the learning process of LLGMN, 20 EMG patterns were extracted from EMG signals for each operation, and the teacher signals consisted of $K \times 20$ patterns, where $K$ is the number of operations. In addition, the operation threshold $\alpha$ was set as 30, the threshold $M_{d}$ as 0.6 , and the threshold $E_{d}$ as 0.2 .

Also, we implemented the signal conversion part on a development board (Xtreme DSP Development Kit-2, Nallatech), which hosts a Xilinx Virtex family FG676 FPGA chip (XCV3000-4FG676). The signal conversion circuit was 


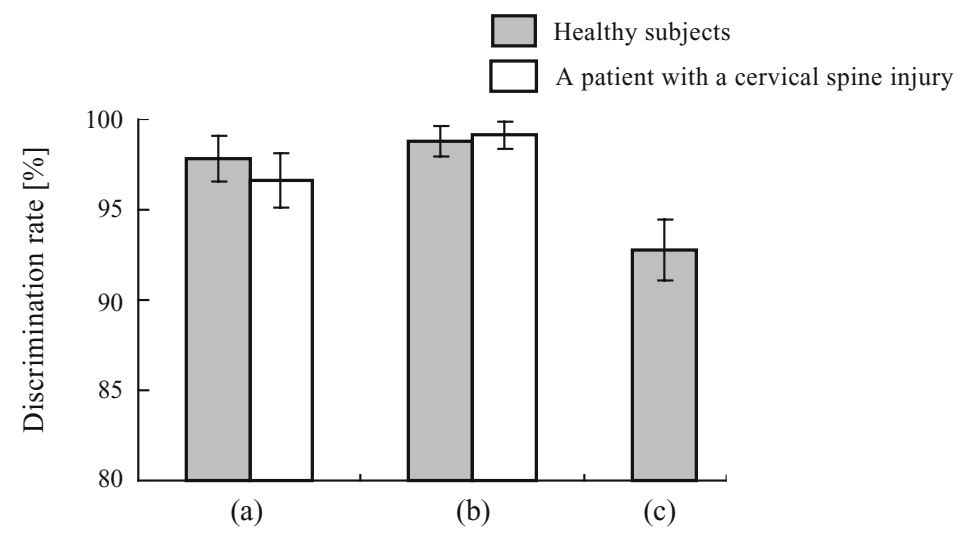

EMG measurement locations :

(a) : Depressor anguli oris; (b) : Zygomaticus major; (c) : Forearm muscles

Fig. 4. EMG pattern discrimination rates

described in Verilog-HDL. The game machine was PS2 (Sony Computer Entertainment Inc.), and the "Othello" game (SUCCESS Corp.) was used for experiments, which can be classified into Type 4 (see Table 1). In this setup, five commands are necessary for operation: up, down, left, right, and stone arrangement.

\subsection{EMG Discrimination Experiments}

The EMG patterns measured during users' operation were discriminated using LLGMN. In the experiments using depressor anguli oris muscles and zygomaticus major muscles, three motions ( $K=3$; contracting muscles on the right side, the left side, and both sides) were discriminated. For forearm muscles, the classes were five motions ( $K=5$; right hand: flexion, extension, grasping, and opening; left hand: grasping). The EMG pattern discrimination rates for three measurement locations are shown in Fig. 4 It can be seen that all of the discrimination rates are in a relatively high level. Using LLGMN, high discrimination accuracy can be achieved, and it is applicable for practical game operation, even if the experimental conditions such as the number of operation and measurement positions were changed.

\subsection{Operation Experiments}

In the experiments, subjects were asked to follow a predefined route and place the stone at a goal (see Fig. 51). EMG signals were measured from depressor anguli oris muscles (see Fig. 3(a)), and three operations can be discriminated $(K=3)$ : contracting muscles on the right side, the left side, and both sides. In this experiment, the shift-group protocol was used for game control, because the number of operations was less than that of commands. Command groups are shown in Fig. 6. In this figure, operation 1 means contracting muscles on 


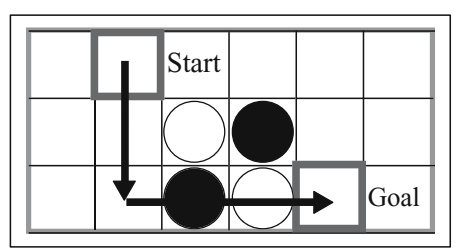

Fig. 5. The desired route

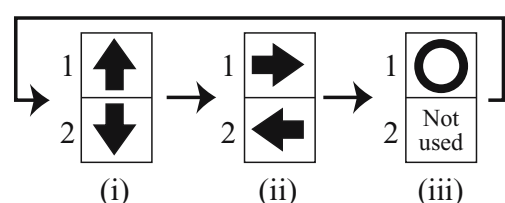

Fig. 6. Command groups

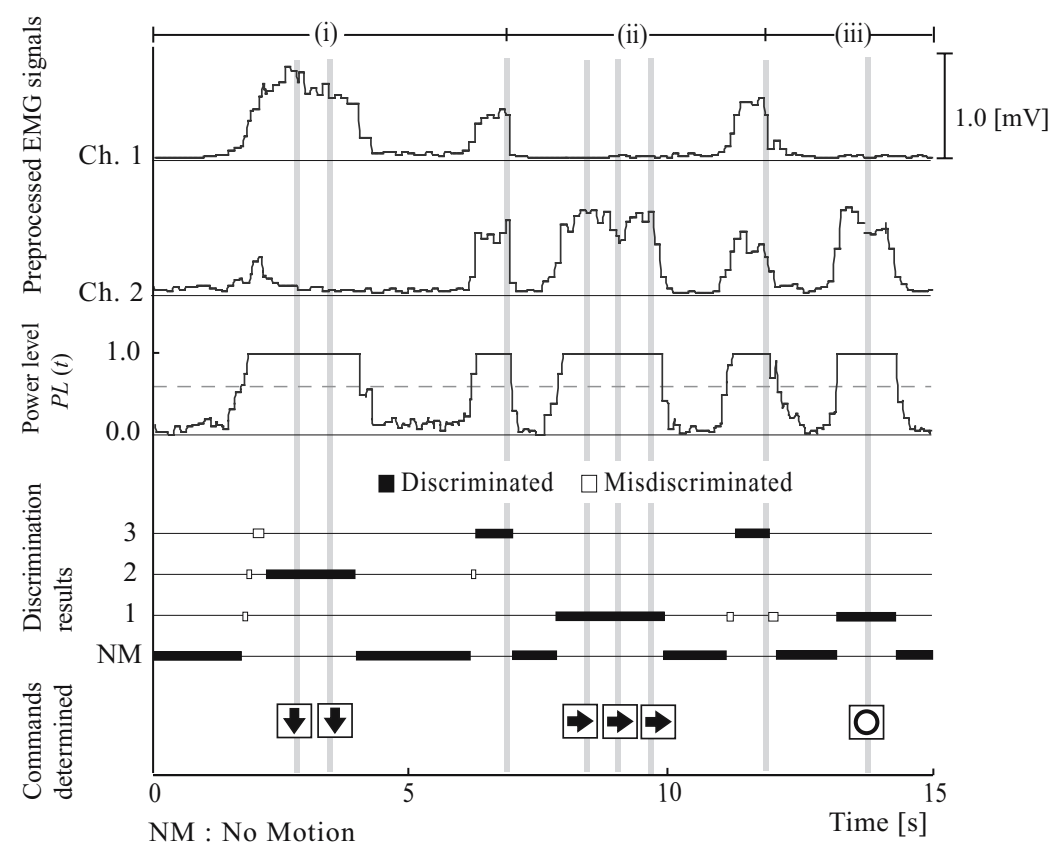

Fig. 7. An example of operations using EMG measured from depressor anguli oris muscles (Subject E)

the right side, operation 2 means contracting muscles on the left side. Also, by contracting muscles on both sides users can shift groups of commands. With this method, five commands can be selected using only three operations.

An example of operations by subject $\mathrm{E}$ is shown in Fig. 7 In this figure, two channels of the preprocessed EMG signals, the power level $P L(t)$, the discrimination results, and control commands are plotted. The gray areas indicate the operations the subject made in the Othello game. The discrimination results were set as no motion (NM) when the power level $P L(t)$ was less than $M_{d}$.

The EMG signals corresponding to the users' operation were discriminated by LLGMN. In this method, misdiscrimination can be reduced using the power level $P L(t)$ and the entropy $E(t)$. However, during the transient phases of users' operations, some misdiscrimination can still be found. Commands corresponding to the users' operations are decided when the same discrimination continues $\alpha$ 


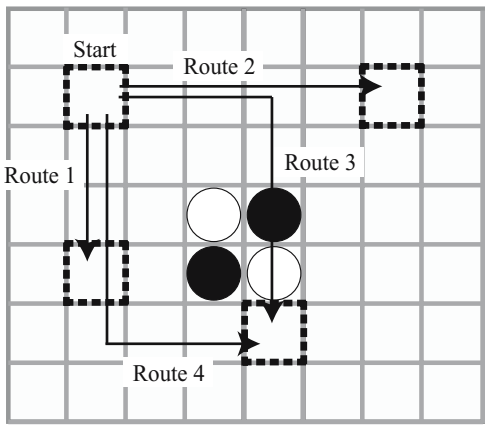

Fig. 8. Four routes used for evaluation experiments
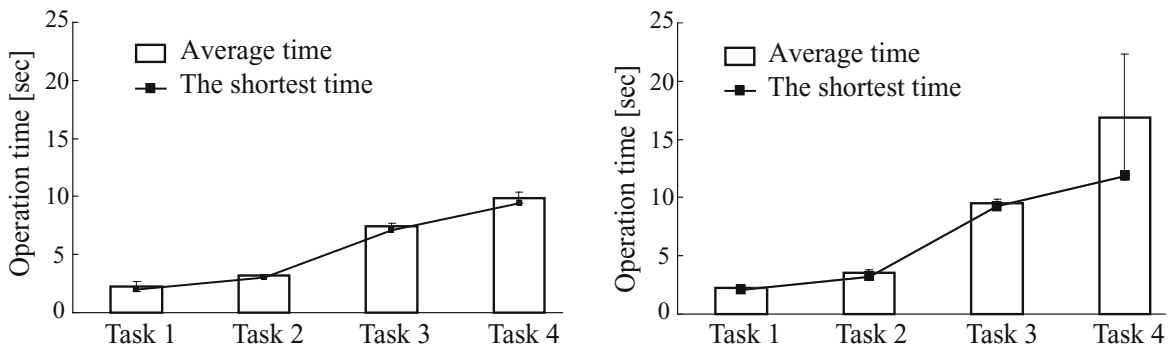

(a) Operation time by healthy subjects (b) Operation time by a patient with a cer(Subjects A-D). vical spine injury (Subject E).

Fig. 9. Comparison of operation time

times. Using this rule, a stable operation can be performed. In this experiment, the commands were selected by operations 1 and 2, and the command groups were shifted by operation 3 . It should also be mentioned that since the shiftgroup method was utilized, some additional commands can be added. From the experimental results, it can be confirmed that the proposed system can work according to subject's intention even when the number of operations were very small.

Then, evaluation experiments were conducted with four predefined routes (see Fig. 8). Subjects were asked to perform these four tasks, and the operation times were recorded. In tasks 1 to 4 , a cursor is moved from the start to three different goals, and in task 4, users are asked to arrange stones on target positions. For each task five trials were performed.

Comparison of operation time in video game operation experiments using depressor anguli oris muscles is shown in Fig. 9, The command groups were set as Fig. 6] In Fig. 9, the bar graph indicates the average operation time, and the sequential line graph indicates the shortest time for each task. It can be seen that, for both healthy subjects and subject E, average time and the shortest time increase when the complexity of the task increases. For subject E, the operation time of task 4 has a large standard deviation. The cursor path in 


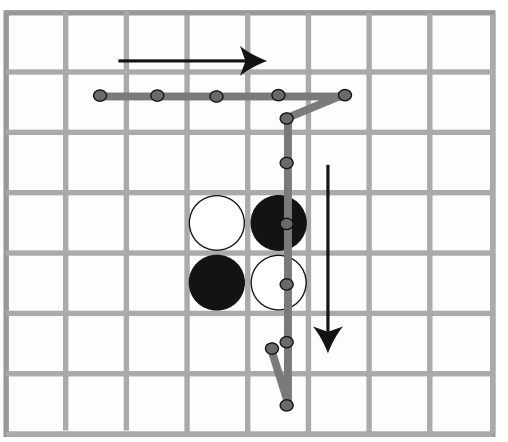

Fig. 10. The most time-consuming trial by Subject E (Task 4)

the most time-consuming trial is plotted in Fig. 10. The cursor control took a long time, because he could not stop at the desired position in this trial. This problem could be related to how to set the parameters such as $M_{d}, E_{d}$, and $\alpha$.

In the experiments, it was confirmed that all subjects could conduct tasks successfully. Further insights are necessary focusing on parameter setting according to the user's profile.

\section{Conclusion}

In this paper, we have proposed a novel entertainment interface using biological signals, and a prototype system has been constructed. In this interface system, a variety of biological signals can be used as input, and users can choose input signals with respect to their conditions, so that a lot more people are able to enjoy video games. Also, the users' intention of operation is estimated from the input signals using a PNN. Due to the adaptive learning capability of neural networks, a high level of discrimination accuracy is achieved. Moreover, video game machines can be easily changed, so that various amusement machines can be incorporated into the proposed system.

To verify validity of the proposed interface, EMG discrimination experiments and video game operation experiments have been carried out with five subjects. In the experiments, discrimination rates of all subjects were about over $90 \%$. With this interface system, subjects can operate games at will.

The game "Othello" used in the experiments is classified into Type 4, and the operations are comparatively easy. In cases of Type 1 games, the proposed method may have some limitations, because Type 1 games are very fast paced and require a variety of complex commands. Thus, it will be necessary to first confirm the feasibility of the proposed method with those games. Also, in future research, we would like to improve the decision algorithm of the operation parameters, and apply the proposed method to other input signals and other amusement machines. 


\section{References}

1. J. Malec, R. Jones, N. Rao, and K. Stubbs, "Video game practice effects on sustained attention in patients with craniocerebral trauma," Cognitive Rehabilitation, Vol. 2, No. 4, pp. 18-23, 1984.

2. R.J. Taylor, and E. Berry, "The use of a computer game to rehabilitate sensorimotor functional deficits following a subarachnoid haemorrhage," Neuropsychological Rehabilitation, Vol. 8, No. 2, pp. 113-122, 1998.

3. K. Coleman, "Electromyography based human-computer-interface to induce movement in elderly persons with movement impairments," Workshop on Universal Accessibility of Ubiquitous Computing: Providing for the Elderly, pp. 75-79, 2001.

4. M. Suzuki, Y. Niida, G. Kamiyama, S. Yamashita, Y. Shiota, T. Tamagaki, and E. Ito, "The interface of a consumer game for a handicapped parson," Transactions of the Japanese Society for Medical and Biological Engineering, Vol. 42, p. 140, 2004. (In Japanese)

5. J.B. Lopes, "Designing User Interfaces for Severely Handicapped Persons," IEEE Transactions on Automatic Control, Vol. 23, No. 4, pp. 538-544, 2001.

6. R. Krepki, B. Blankertz, G. Gurio, and K.R. Muller, "The Berlin Brain-Computer Interface (BBCI)," IEEE Transactions on Automatic Control, Vol. 23, No. 4, pp. 538-544, 2003.

7. M. Betke, J. Gips, and P. Fleming, "The Camera Mouse: Visual Tracking of Body Features to Provide Computer Access for People With Severe Disabilities," IEEE Transactions on Neural System and Rehabilitation Engineering, Vol. 10, No. 1, pp. 1-10, 2002.

8. D. Specht, "Probabilistic Neural Networks," Neural Networks, Vol. 3, No. 1, pp. 109-118, 1990.

9. A. Hiraiwa, K. Shimohara, and Y. Tokunaga, "EMG pattern analysis and classification by neural network," Proceedings of IEEE International Conference on Systems, Man and Cybernetics, pp. 1113-1115, 1989.

10. A. Hiraiwa, U. Uchida, and K. Shimohara, "EMG/EEG pattern recognition by neural networks," Proceedings of the Eleventh European Meeting on Cybernetics and Systems Research, pp. 1383-1390, 1992.

11. M.F. Kelly, P.A. Parker, and R.N. Scott, "The Application of Neural Networks to Myoelectric Signal Analysis A Preliminary Study," IEEE Transactions on Biomedical Engineering, Vol. 37, No. 3, pp. 221-230, 1990.

12. T. Tsuji, O. Fukuda, H. Ichinobe, and M. Kaneko, "A Log-Linearized Gaussian Mixture Network and Its Application to EEG Pattern Classification," IEEE Transactions on Systems, Man, and Cybernetics-Part C: Applications and Reviews, Vol. 29, No. 1, pp. 60-72, 1999.

13. T. Tsuji, N. Bu, M. Kaneko, and O. Fukuda, "A Recurrent Log-linearized Gaussian Mixture Network," IEEE Transactions on Neural Networks, Vol. 14, No. 2, pp. 304-316, 2003.

14. T. Tsuji, H. Ichinobe, K. Ito, and M. Nagamachi, "Discrimination of Forearm Motions from EMG Signals by Error Back Propagation Typed Neural Network Using Entropy," Transactions of the Society of Instrument and Control Engineers, Vol. 29CNo. 10Cpp. 1213-1220, 1993. (In Japanese)

15. S. Shitamori, T. Tsuji, O. Fukuda, Y. Uchida, and N. Mitoda, "Development of Barrier Free Interface: BIO-REMOTE," SICE System Integration Division Annual Conference, pp. 415-416, 2002. (In Japanese) 